International Journal of Difference Equations (IJDE).

ISSN 0973-6069, Volume 15, Number 1, (2020). 45-70

(C) Research India Publications

https://dx.doi.org/10.37622/IJDE/15.1.2020.45-70

\title{
Oscillatory Behaviour of Higher Order Neutral Differential Equations with Several Delays and with a Super Linear Term
}

\author{
Kali Charan Panda ${ }^{1}$, Radhanath Rath ${ }^{* 2}$, and Subhendu Kumar Rath ${ }^{3}$ \\ ${ }^{1}$ Department Of Mathematics, Triddent Academy of Technology, Bhubaneswar,

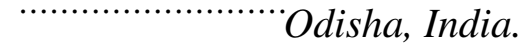 \\ ${ }^{2}$ Former Professor (VSSUT Burla, 768018) and Retired Principal, (Khallikote \\ Autonomous College, Berhampur, 760001). \\ Sailashree vihar, Centerpoint apartment, Flat A-203, Near C. S Pur P.S, Bhubaneswar,

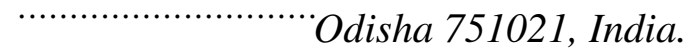 \\ ${ }^{3}$ Deputy Registrar, BPUT, Rourkella, Odisha, India.
}

\begin{abstract}
This article, is concerned with finding sufficient conditions for the oscillatory and asymptotic behaviour of solutions of the higher order neutral delay differential equation

$$
\left(y(t)-\sum_{j=1}^{k} p_{j}(t) y\left(r_{j}(t)\right)\right)^{(n)}+v(t) G(y(g(t)))-u(t) H(y(h(t)))=f(t)
$$

where, $n \geq 2$, is any positive integer, $p_{i}, f \in C([0, \infty), \mathbb{R}), r_{i}, g, h, v, u \in$ $C([0, \infty),[0, \infty)), G$ and $H \in C(\mathbb{R}, \mathbb{R})$. The functional delays $r_{j}(t) \leq t$ for each $j$ and $g(t) \leq t, h(t) \leq t$ and all of them approach $\infty$ as $t \rightarrow \infty$.The results hold when $u \equiv 0$ and $f(t) \equiv 0$. This paper extends, generalizes and improves some known results and answers an open problem.
\end{abstract}

Key words and phrases: Oscillatory solution; nonoscillatory solution; asymptotic behaviour, neutral equation.

2000 Mathematics Subject Classification: 34C10, 34C15, 34K40

\footnotetext{
${ }^{*}$ Corresponding Author
} 


\section{INTRODUCTION}

This article, is concerned with finding sufficient conditions for the oscillatory and asymptotic behaviour of solutions of the higher order neutral delay differential equation (NDDE in short)

$$
\left(y(t)-\sum_{j=1}^{k} p_{j}(t) y\left(r_{j}(t)\right)\right)^{(n)}+v(t) G(y(g(t)))-u(t) H(y(h(t)))=f(t),
$$

where, $n \geq 2$ is any positive integer, $p_{j}, r_{j} \in C^{(n)}([0, \infty), \mathbb{R})$ and $p_{j}$ are bounded for each $j=1,2, \ldots, k$. Further, $f \in C([0, \infty), \mathbb{R}), g, h, v, u \in C([0, \infty),[0, \infty)), G$ and $H \in C(\mathbb{R}, \mathbb{R})$. The functional delays, each $r_{j}(t) \leq t, g(t) \leq t$ and $h(t) \leq t$ and all of them approach $\infty$ as $t \rightarrow \infty$. The results hold when $u \equiv 0, f(t) \equiv 0$, and $G(u) \equiv u$.

Some of the following assumptions would be needed later in this article.

$$
\begin{gathered}
x G(x)>0 \text { for } x \neq 0 . \\
v(t)>0, \int_{t_{0}}^{\infty} v(t) d t=\infty .
\end{gathered}
$$

There exists a real valued bounded function $F(t)$ such that $F^{(n)}(t)=f(t)$.

The function $F(t)$ in (1.4) satisfies $\lim _{t \rightarrow \infty} F(t)=0$.

$$
\int_{t_{0}}^{\infty} t^{n-1} u(t) d t<\infty
$$

$H$ is bounded and $x H(x)>0$ for $x \neq 0$.

$$
\begin{gathered}
\int_{t_{0}}^{\infty} t^{n-1} v(t) d t=\infty . \\
\liminf _{t \rightarrow \infty} g(t) / t>0 .
\end{gathered}
$$

For $u>0$ there exists $\delta>0$ such that $G(u) \geq \delta u$ and for $u<0$ there exists $\delta>0$

$$
\begin{gathered}
\text { such that } G(u) \leq \delta u . \\
\int_{t_{0}}^{\infty} t^{n-2} v(t)=\infty \text { for } n \geq 2 .
\end{gathered}
$$

Remark 1.1. Note that $\int_{0}^{\infty} t^{n-1}|f(t)| d t<\infty$, if and only if (1.4) and (1.5) hold.

We assume $p_{j}(t), j=1,2, \ldots, k$, to be bounded and satisfy one of the following conditions.

$$
p_{j}(t) \geq 0, \text { for every } j=1,2, \ldots k \text { and } \sum_{j=1}^{k} \limsup _{t \rightarrow \infty} p_{j}(t)<p<1 .
$$




$$
p_{j}(t) \leq 0, \text { for every } j=1,2, \ldots k \text { and } \sum_{j=1}^{k} \liminf _{t \rightarrow \infty} p_{j}(t)>-p>-1 \text {. }
$$

$p_{j}(t)<0$ for every $j=1,2, \ldots k$ and there exists, $i \in\{1,2,3, \ldots, k\}$ such that

$$
\limsup _{t \rightarrow \infty} p_{i}(t)-\sum_{j \neq i} \liminf _{t \rightarrow \infty} p_{j}(t)<-1 \text {. }
$$

$p_{j}(t)>0$ for every $j=1,2, \ldots k$ and there exists, $i \in\{1,2,3, \ldots, k\}$ such that

$$
\begin{gathered}
\liminf _{t \rightarrow \infty} p_{i}(t)-\sum_{j \neq i} \limsup _{t \rightarrow \infty} p_{j}(t)>1 . \\
-b \leq p_{j}(t) \leq 0, \text { for } j=1,2, \ldots, k \\
0 \leq p_{j}(t) \leq b \text { for } j=1,2, \ldots, k .
\end{gathered}
$$

Remark 1.2. If $p_{i}(t)=p(t)$ and $p_{j}(t)=0$, for $j \neq i$, then the conditions (1.12) (1.17) due to the boundedness of $p_{j}(t)$ reduces to the following conditions

(A1): $0 \leq p(t) \leq p<1, \quad(\mathrm{~A} 2):-1<-p \leq p(t) \leq 0,(\mathrm{~A} 3):-p_{1} \leq p(t) \leq-p<-1$ (A4): $p_{1} \geq p(t) \geq p>1 \quad(\mathrm{~A} 5):-b \leq p(t) \leq 0 \quad$ (A6): $0 \leq p(t) \leq b$

respectively where $p, p_{1}$ and $b$ are positive scalars. These conditions are assumed in $[9,11,12,13]$ to study qualitative behaviour of solution of (1.18).

\section{Motivation and objective}

In recent years, a great deal of research work is done on oscillation theory of neutral delay differential equations( see $[3,9,11,12,14,15,17]$ and the references cited therein). The authors [12] have found oscillation criteria for the NDDE

$$
(y(t)-p(t) y(t-\tau))^{(n)}+q(t) G(y(t-\sigma))=f(t),
$$

where $p(t)$ satisfies one of the conditions (A1)-(A4) as mentioned in remark 1.2. Recently, in [3] the authors obtained the oscillatory and asymptotic behaviour of the solutions of the NDDE

$$
(y(t)-p(t) y(t-\tau))^{(n)}+q(t) G(y(t-\sigma))-u(t) H(y(t-\alpha)=f(t) .
$$

In literature we find that (1.1) is very rarely studied. In this article by following the suggestion of Gyori and Ladas in an open problem [1][Notes 1.8, page 31] we extend 
the lemma [1][lemma 1.5.2] suitably for its own sake and its application to the study of the oscillatory and asymptotic behavior of solutions of neutral differential equation (1.1) with multiple delays under the derivative sign. As, we could relax the condition " $G$ is non decreasing"in this paper, we have substantially improved and generalized the results of [3] and [11] and further answered another open problem [1][problem 10.10.2, page 287]. Note that [1][lemma 1.5.2] was used in the papers [3,11].

"Let $t_{1}$ be a fixed positive real number and

$$
t_{0}=\min \left\{\inf _{t \geq t_{1}}\left(r_{1}(t), r_{2}(t), \ldots, r_{k}(t)\right), \inf _{t \geq t_{1}} g(t), \inf _{t \geq t_{1}} h(t)\right\}
$$

A function $y \in C\left(\left[t_{0}, \infty\right), \mathbb{R}\right)$ such that $y(t)-\sum_{i=1}^{k} p_{i}(t) y\left(r_{i}(t)\right)$ is $n$ times continuously differentiable on $\left[t_{0}, \infty\right)$ and which satisfies the neutral equation (1.1) for all $t \geq t_{1}$, is defined as a solution of (1.1). This solution of (1.1) is called unique, if there exists an initial function $\phi \in C\left(\left[t_{0}, t_{1}\right], \mathbb{R}\right)$ such that $y(t)=\phi(t)$ for all $t \in\left[t_{0}, t_{1}\right]$. A solution is called positive or eventually positive if one finds a real $t_{1}$ such that $y(t)>0$ for $t \in\left(t_{1}, \infty\right)$. Similarly a solution is called negative or eventually negative if $y(t)<0$ in $\left(t_{1}, \infty\right)$ for some $t_{1}$. A solution which is neither positive nor negative is called oscillatory. Note that, a solution of (1.1) is also said to be oscillatory if it has arbitrary large zeros, otherwise called non oscillatory."

\section{SOME LEMMAS}

Some lemmas that would be used in the sequel, are going to be presented in this section.

Lemma 2.1. [16] "Let $u(t)$ and $v(t)$ be two real valued continuous functions defined for $t \geq t_{0} \geq 0$. Then

$$
\begin{aligned}
& \lim \inf _{t \rightarrow \infty} u(t)+\lim \inf _{t \rightarrow \infty} v(t) \\
\leq & \lim \inf _{t \rightarrow \infty}(u(t)+v(t)) \\
\leq & \lim \sup _{t \rightarrow \infty} u(t)+\lim \inf _{t \rightarrow \infty} v(t)\left(\text { or } \lim \inf _{t \rightarrow \infty} u(t)+\lim \sup _{t \rightarrow \infty} v(t)\right) \\
\leq & \lim \sup _{t \rightarrow \infty}(u(t)+v(t)) \\
\leq & \lim \sup _{t \rightarrow \infty} u(t)+\lim \sup _{t \rightarrow \infty} v(t)
\end{aligned}
$$

provided that no sum is of the form $\infty-\infty$. "

Lemma 2.2. [16] "Let $u(t)$ and $v(t)$ be two non negative real valued continuous 
functions defined for $t \geq t_{0}$. Then

$$
\begin{aligned}
& \lim \inf _{t \rightarrow \infty} u(t) \times \lim \inf _{t \rightarrow \infty} v(t) \\
\leq & \lim \inf _{t \rightarrow \infty}(u(t) \times v(t)) \\
\leq & \lim \sup _{t \rightarrow \infty} u(t) \times \lim \inf _{t \rightarrow \infty} v(t)\left(\text { or } \lim \inf _{t \rightarrow \infty} u(t) \times \lim \sup _{t \rightarrow \infty} v(t)\right) \\
\leq & \lim \sup _{t \rightarrow \infty}(u(t) \times v(t)) \\
\leq & \lim \sup _{t \rightarrow \infty} u(t) \times \lim \sup _{t \rightarrow \infty} v(t)
\end{aligned}
$$

provided that no product is of the form $0 \times \infty$."

Lemma 2.3. [4, p.193] "Let $y \in C^{n}([0, \infty), \mathbb{R})$ be of constant sign and $\not \equiv 0$ in any interval $[T, \infty), T \geq 0$, and $y^{(n)}(t) y(t) \leq 0$.Then there exists a number $t_{0} \geq 0$ such that the functions $y^{(j)}(t), j=1,2, \ldots, n-1$, are of constant sign on $\left[t_{0}, \infty\right)$ and there exists a number $m \in\{1,3, . ., n-1\}$ when $n$ is even or $m \in\{0,2,4, \ldots, n-1\}$ when $n$ is odd such that

$$
\begin{gathered}
y(t) y^{(j)}(t)>0, \text { for } j=0,1,2, \ldots, m, t \geq t_{0}, \\
(-1)^{n+j-1} y(t) y^{(j)}(t)>0 \text { for } j=m+1, m+2, \ldots, n-1, t \geq t_{0} .
\end{gathered}
$$

Lemma 2.4. [5, p.193] If $f$ and $g$ be two positive functions in $[a, t]$ and $\lim _{t \rightarrow \infty} \frac{f(t)}{g(t)}=$ $l \in R$, where l is non-zero, then $\int_{a}^{\infty} f(t) d t$ and $\int_{a}^{\infty} g(t) d t$ converge or diverge together. Also, if $f / g \rightarrow 0$ and $\int_{a}^{\infty} g(t) d t$ converges, then $\int_{a}^{\infty} f(t) d t$ converges and if $f / g \rightarrow \infty$ and $\int_{a}^{\infty} g(t) d t$ diverges, then $\int_{a}^{\infty} f(t) d t$ diverges.

The following lemma generalizes [1][Lemma 1.5.2].

Lemma 2.5. [17] "Suppose that $\tau(t)$ is a continuous and strictly increasing unbounded function such that $\tau(t) \leq t$. Let $u, v, p:\left[t_{0}, \infty\right) \rightarrow R$ be such that

$$
u(t)=v(t)-p(t) v(\tau(t)), \quad t \geq \tau_{-1}\left(t_{0}\right) .
$$

Suppose that $p(t)$ is in one of the ranges

$$
\begin{array}{r}
0 \leq p(t) \leq p_{1}, \\
-1<-p \leq p(t) \leq 0,
\end{array}
$$

or

$$
-p_{2} \leq p(t) \leq-p_{1}<-1,
$$

where $p, p_{1}, p_{2}$ are positive real numbers. If $v(t)>0$ for $t \geq t_{0}>0$ and $\liminf _{t \rightarrow \infty} v(t)=0$ and $\lim _{t \rightarrow \infty} u(t)=L \in R$ exists, then $L=0$. ” 


\section{MAIN RESULTS-I}

By following the suggestion in [1][note 1.8, page 31], we now extend the above lemma from single functional delay to several functional delays for its application to study the qualitative behaviour of solutions of (1.1).

Lemma 3.1 (Extended Gyori Ladas Lemma-I). Suppose that, for each $i=$ $1,2, \ldots, k, p_{i}, r_{i} \in C\left(\left[t_{0}, \infty\right), \mathbb{R}\right), p_{i}$ are bounded, $r_{i}(t) \leq t$ and $\lim _{t \rightarrow \infty} r_{i}(t)=\infty$. Further, suppose that $y \in C\left(\left[t_{0}, \infty\right), \mathbb{R}\right)$. Assume $y(t)>0$ for $t \geq t_{0}$. Let

$$
z(t)=y(t)-\sum_{j=1}^{k} p_{j}(t) y\left(r_{j}(t)\right), \quad t \geq t_{1}>t_{0} .
$$

If $\liminf _{t \rightarrow \infty} y(t)=0$ and $\lim _{t \rightarrow \infty} z(t)=\delta \in \mathbb{R}$ exists then the following statements are true.

(a) If $p_{j}(t) \geq 0$ for each $j$ then $\delta \leq 0$ and $p_{j}(t) \leq 0$ for each $j$ then $\delta \geq 0$.

(b) Further, suppose that $y(t)$ is bounded and $p_{j}(t), j=1,2, \ldots, k$, satisfy one of the four conditions (1.12),(1.13),(1.14) or(1.15). Then $\delta=0$ and $\lim _{t \rightarrow \infty} y(t)=0$.

Proof. (a) Since $\lim _{t \rightarrow \infty} z(t)=\delta$ exists finitely then $\liminf _{t \rightarrow \infty} z(t)=$ $\lim \sup _{t \rightarrow \infty} z(t)=\delta$. If $p_{j}(t) \geq 0$ then $z(t) \leq y(t)$ and $\liminf _{t \rightarrow \infty} z(t) \leq$ $\liminf _{t \rightarrow \infty} y(t)$. This implies $\delta \leq 0$. Again if $p_{j}(t) \leq 0$ then $z(t) \geq y(t)$ and this implies $\delta \geq 0$. Hence the result follows.

(b) Consider case (i) i.e; suppose $p_{j}(t)$ satisfy (1.12). As $p_{i}(t) \geq 0$, by part (a) above $\delta \leq 0$. Then applying lemma2.1 and 2.2 we have 


$$
\begin{aligned}
0 \geq \delta & =\limsup _{t \rightarrow \infty} z(t)=\limsup _{t \rightarrow \infty}\left(y(t)-\sum_{j=1}^{k} p_{j}(t) y\left(r_{j}(t)\right)\right) \\
& \geq \limsup _{t \rightarrow \infty} y(t)+\liminf _{t \rightarrow \infty}\left(-\sum_{j=1}^{k} p_{j}(t) y\left(r_{j}(t)\right)\right) \\
& \geq \limsup _{t \rightarrow \infty} y(t)-\limsup _{t \rightarrow \infty}\left(\sum_{j=1}^{k} p_{j}(t) y\left(r_{j}(t)\right)\right) \\
& \geq \limsup _{t \rightarrow \infty} y(t)-\sum_{j=1}^{k} \limsup _{t \rightarrow \infty}\left(p_{j}(t) y\left(r_{j}(t)\right)\right) \\
& \geq \limsup _{t \rightarrow \infty} y(t)-\sum_{j=1}^{k} \limsup _{t \rightarrow \infty} p_{j}(t) \limsup y\left(r_{j}(t)\right) \\
& \geq \limsup _{t \rightarrow \infty} y(t)\left(1-\sum_{j=1}^{k} \limsup _{t \rightarrow \infty} p_{j}(t)\right) . \\
& \geq \limsup _{t \rightarrow \infty} y(t)(1-p) \geq 0 .
\end{aligned}
$$

Hence $\delta=0$ and $\lim \sup _{t \rightarrow \infty} y(t)=0$ by (1.12). Then $\lim _{t \rightarrow \infty} y(t)=0$.

Next consider case ii i.e; $p_{j}(t)$ satisfy(1.13). Clearly, $z(t) \geq 0$ due to (1.13) and this implies $\delta \geq 0$. Application of lemmas 2.1 and 2.2 to (3.1) yields :

$$
\begin{aligned}
\delta=\liminf _{t \rightarrow \infty} z(t) & =\liminf _{t \rightarrow \infty}\left(y(t)-\sum_{j=1}^{k} p_{j}(t) y\left(r_{j}(t)\right)\right) \\
& \leq \liminf _{t \rightarrow \infty} y(t)+\limsup _{t \rightarrow \infty}\left(\sum_{j=1}^{k}-p_{j}(t) y\left(r_{j}(t)\right)\right) \\
& \leq \sum_{j=1}^{k} \limsup _{t \rightarrow \infty}\left(-p_{j}(t)\right) \limsup _{t \rightarrow \infty}\left(y\left(r_{j}(t)\right)\right) \\
& =\sum_{j=1}^{k}-\liminf _{t \rightarrow \infty}\left(p_{j}(t) \limsup _{t \rightarrow \infty}\left(y\left(r_{j}(t)\right)\right)\right. \\
& \leq p \limsup _{t \rightarrow \infty}(y(t)) \leq p \alpha .
\end{aligned}
$$

Hence we get,

$$
\alpha \geq \frac{\delta}{p}>\delta
$$


Again

$$
\begin{aligned}
\delta=\limsup _{t \rightarrow \infty} z(t) & =\limsup _{t \rightarrow \infty}\left(y(t)-\sum_{j=1}^{k} p_{j}(t) y\left(r_{j}(t)\right)\right) \\
& \geq \limsup _{t \rightarrow \infty} y(t)+\liminf _{t \rightarrow \infty}\left(\sum_{j=1}^{k}-p_{j}(t) y\left(r_{j}(t)\right)\right) \\
& \geq \limsup _{t \rightarrow \infty} y(t)+\sum_{j=1}^{k} \liminf _{t \rightarrow \infty}\left(\left(-p_{j}(t)\right) y\left(r_{j}(t)\right)\right) \\
& \geq \limsup _{t \rightarrow \infty} y(t)+\sum_{j=1}^{k} \liminf _{t \rightarrow \infty}\left(-p_{j}(t)\right) \liminf _{t \rightarrow \infty} y\left(r_{j}(t)\right) \\
& =\limsup _{t \rightarrow \infty} y(t)=\alpha
\end{aligned}
$$

From this and (3.2) it follows that

$$
\alpha>\delta \geq \alpha
$$

a contradiction. This implies $\delta=0=\alpha$. Then $\lim _{t \rightarrow \infty} y(t)=0$.

Next consider case iii:i.e; $p_{j}(t)$ satisfy(1.14). Then proceeding with the application of lemmas 2.1 and 2.2 to (3.1) we obtain

$$
\begin{aligned}
\delta=\liminf _{t \rightarrow \infty} z(t) & =\liminf _{t \rightarrow \infty}\left(y(t)-\sum_{j=1}^{k} p_{j}(t) y\left(r_{j}(t)\right)\right) \\
& \leq \limsup _{t \rightarrow \infty}\left(y(t)+\sum_{j \neq i}-p_{j}(t) y\left(r_{j}(t)\right)\right)+\liminf _{t \rightarrow \infty}\left(-p_{i}(t) y\left(r_{i}(t)\right)\right) \\
& \leq \limsup _{t \rightarrow \infty} y(t)+\limsup _{t \rightarrow \infty} \sum_{j \neq i}-p_{j}(t) y\left(r_{j}(t)\right)+\limsup _{t \rightarrow \infty}\left(-p_{i}(t)\right) \liminf _{t \rightarrow \infty}\left(y\left(r_{i}(t)\right)\right) \\
& \leq \limsup _{t \rightarrow \infty} y(t)+\sum_{j \neq i} \limsup _{t \rightarrow \infty}\left(\left(-p_{j}(t)\right) y\left(r_{j}(t)\right)\right) \\
& \leq \limsup _{t \rightarrow \infty} y(t)+\sum_{j \neq i} \limsup _{t \rightarrow \infty}\left(-p_{j}(t)\right) \limsup _{t \rightarrow \infty}\left(y\left(r_{j}(t)\right)\right) \\
& \leq \limsup _{t \rightarrow \infty}(y(t))\left[1-\sum_{j \neq i} \liminf _{t \rightarrow \infty} p_{j}(t)\right] .
\end{aligned}
$$


Again we have

$$
\begin{aligned}
\delta=\limsup _{t \rightarrow \infty} z(t) & =\limsup _{t \rightarrow \infty}\left(y(t)-\sum_{j=1}^{k} p_{j}(t) y\left(r_{j}(t)\right)\right) \\
& \geq \liminf _{t \rightarrow \infty} y(t)+\limsup _{t \rightarrow \infty}\left(\sum_{j=1}^{k}-p_{j}(t) y\left(r_{j}(t)\right)\right) \\
& \geq \limsup _{t \rightarrow \infty}\left(-p_{i}(t) y\left(r_{i}(t)\right)+\liminf _{t \rightarrow \infty} \sum_{j \neq i}\left(-p_{j}(t) y\left(r_{j}(t)\right)\right)\right. \\
& \geq \limsup _{t \rightarrow \infty} y\left(r_{i}(t)\right) \liminf _{t \rightarrow \infty}\left(-p_{i}(t)\right)+\sum_{j \neq i} \liminf _{t \rightarrow \infty}\left(\left(-p_{j}(t)\right) y\left(r_{j}(t)\right)\right) \\
& \geq \limsup _{t \rightarrow \infty} y(t)\left(-\limsup _{t \rightarrow \infty} p_{i}(t)\right)+\sum_{j \neq i} \liminf _{t \rightarrow \infty}\left(-p_{j}(t)\right) \liminf _{t \rightarrow \infty} y\left(r_{j}(t)\right) \\
& \geq \limsup _{t \rightarrow \infty} y(t)\left(-\limsup _{t \rightarrow \infty} p_{i}(t)\right) .
\end{aligned}
$$

From (3.3) and (3.4), it follows that

$$
\limsup _{t \rightarrow \infty} y(t)\left(\sum_{j \neq i} \liminf _{t \rightarrow \infty} p_{j}(t)-1-\limsup _{t \rightarrow \infty} p_{i}(t)\right) \leq 0 .
$$

Using (1.14), we obtain $\alpha=\lim \sup _{t \rightarrow \infty} y(t)=0$. Then $\lim _{t \rightarrow \infty} y(t)=0$ and further, using (3.3) and (3.4) we obtain $\delta=\lim _{t \rightarrow \infty} z(t)=0$.

Next consider case iv:i.e; $p_{j}(t)$ satisfy(1.15). Then proceeding with the application of lemmas 2.1 and 2.2 to (3.1) we obtain

$$
\begin{aligned}
\delta=\liminf _{t \rightarrow \infty} z(t) & =\liminf _{t \rightarrow \infty}\left(y(t)-\sum_{j=1}^{k} p_{j}(t) y\left(r_{j}(t)\right)\right) \\
& \leq \limsup _{t \rightarrow \infty} y(t)+\liminf _{t \rightarrow \infty} \sum_{j=1}^{k}-p_{j}(t) y\left(r_{j}(t)\right) \\
& \leq \limsup _{t \rightarrow \infty} y(t)-\limsup _{t \rightarrow \infty} \sum_{j=1}^{k} p_{j}(t) y\left(r_{j}(t)\right) \\
& \leq \alpha-\liminf _{t \rightarrow \infty} \sum_{j \neq i} p_{j}(t) y\left(r_{j}(t)\right)-\limsup _{t \rightarrow \infty}\left(p_{i}(t)\right)\left(y\left(r_{i}(t)\right)\right) \\
& \leq \alpha-\sum_{j \neq i} \liminf _{t \rightarrow \infty}\left(-p_{j}(t)\right)\left(y\left(r_{j}(t)\right)\right)-\liminf _{t \rightarrow \infty}\left(p_{i}(t)\right) \limsup _{t \rightarrow \infty}\left(y\left(r_{i}(t)\right)\right) \\
& \leq \alpha-\sum_{j \neq i} \liminf _{t \rightarrow \infty} p_{j}(t) \liminf _{t \rightarrow \infty} y\left(r_{j}(t)\right)-\liminf _{t \rightarrow \infty}\left(p_{i}(t)\right) \limsup _{t \rightarrow \infty} y(t) . \\
& \leq \alpha\left(1-\liminf _{t \rightarrow \infty} p_{i}(t)\right)
\end{aligned}
$$


Again we have

$$
\begin{aligned}
\delta=\limsup _{t \rightarrow \infty} z(t) & =\limsup _{t \rightarrow \infty}\left(y(t)-\sum_{j=1}^{k} p_{j}(t) y\left(r_{j}(t)\right)\right) \\
& \geq \liminf _{t \rightarrow \infty} y(t)+\limsup _{t \rightarrow \infty}\left(\sum_{j=1}^{k}-p_{j}(t) y\left(r_{j}(t)\right)\right) \\
& \geq-\liminf _{t \rightarrow \infty}\left(\sum_{j=1}^{k} p_{j}(t) y\left(r_{j}(t)\right)\right) \\
& \geq-\liminf _{t \rightarrow \infty}\left(p_{i}(t) y\left(r_{i}(t)\right)\right)-\limsup _{t \rightarrow \infty} \sum_{j \neq i}\left(p_{j}(t) y\left(r_{j}(t)\right)\right) \\
& \geq-\liminf _{t \rightarrow \infty} y\left(r_{i}(t)\right) \limsup _{t \rightarrow \infty} p_{i}(t)-\sum_{j \neq i} \limsup \left(\left(-p_{j}(t)\right) y\left(r_{j}(t)\right)\right) \\
& \geq-\sum_{j \neq i} \limsup _{t \rightarrow \infty} p_{j}(t) \limsup _{t \rightarrow \infty} y\left(r_{j}(t)\right) \\
& \geq-\limsup _{t \rightarrow \infty} y(t)\left(\sum_{j \neq i} \limsup _{t \rightarrow \infty} p_{j}(t)\right) \\
& =-\alpha\left(\sum_{j \neq i} \limsup _{t \rightarrow \infty} p_{j}(t)\right) .
\end{aligned}
$$

From (3.5) and (3.6), it follows that

$$
-\alpha\left(\sum_{j \neq i} \limsup _{t \rightarrow \infty} p_{j}(t)\right) \leq \delta \leq \alpha\left(1-\liminf _{t \rightarrow \infty} p_{i}(t)\right)
$$

This implies

$$
\alpha\left(1-\liminf _{t \rightarrow \infty} p_{i}(t)+\sum_{j \neq i} \limsup _{t \rightarrow \infty} p_{j}(t)\right) \geq 0 .
$$

By (1.15), we obtain $\alpha \leq 0$. Since $y(t)>0$ then $\alpha=0$. This implies $\lim _{t \rightarrow \infty} y(t)=0$. By (3.6), it follows that $\delta \geq 0$. Using (a), we obtain $\delta=0$. Thus the lemma is proved.

Lemma 3.2 (Extended Gyori Lada Lemma-II). Assume $y(t)<0$ for $t \geq t_{0}$ and $\limsup _{t \rightarrow \infty} y(t)=0$. Suppose that $z(t)$ is defined as in (3.1). Further, assume and $\lim _{t \rightarrow \infty} z(t)=\delta$ exists finitely. Then

(a) If $p_{j}(t) \geq 0$ for each $j$ then $\delta \geq 0$ and $p_{j}(t) \leq 0$ for each $j$ then $\delta \leq 0$.

(b) Further, suppose that $y(t)$ is bounded and $p_{j}(t), j=1,2, \ldots, k$, satisfy one of the conditions (1.12), (1.13), (1.14) or (1.15). Then $\delta=0$ and $\lim _{t \rightarrow \infty} y(t)=0$. 
Proof. Proceeding as in the proof of above lemma with the substitution $x(t)=-y(t)>$ 0 , one may complete the proof of the lemma.

Remark 3.3. Observe that $u(t)$ and $v(t)$ are not assumed to be bounded in Lemmas 2.1 or 2.2. However, we assume that $y(t)$ and $y\left(r_{j}(t)\right)$ are bounded in lemma 3.1. This is done, only to avoid the statement, "provided that no sum is of the form $\infty-\infty$ " in Lemma 2.1 and, "provided that no product is of the form $0 \times \infty$ " in Lemma 2.2. However, if $p_{j}(t)$ satisfies (1.13) or (1.14), then the terms in $z(t)$ are positive when $y(t)>0$. Hence in the limiting case the sum cannot yield $\infty-\infty$ form. Further, if $\liminf _{t \rightarrow \infty} p_{j}(t)>0$ for each $j$ in the case when $p_{j}(t)$ satisfies (1.13) then the product $-p_{j}(t) y\left(r_{j}(t)\right)$ in the limiting case cannot be of the form $0 \times \infty$. Thus, if $p_{j}(t)$ satisfies (1.13) or (1.14), we can relax the condition of boundedness on $y(t)$ in the lemma 3.1. We state this as a lemma.

Lemma 3.4 (Extended Gyori Lada Lemma-III). Assume $y(t)>0$ for $t \geq t_{0}$ with $\liminf _{t \rightarrow \infty} y(t)=0$, and let $z(t)$ be defined as in (3.1). Assume $\lim _{t \rightarrow \infty} z(t)=\delta$ exists is finite. Let $p_{j}(t)$ satisfy (1.13) or (1.14). Assume $\liminf _{t \rightarrow \infty}\left|p_{j}(t)\right|>0$ for the case $p_{j}(t)$ satisfying (1.13). Then $\delta=0$ and $\lim _{t \rightarrow \infty} y(t)=0$.

Before the last lemma in this section is stated, it is assumed that $y(t)$ is a non-oscillatory solution of (1.1) for $t \geq t_{1}$. Define for $t \geq t_{1}$,

$$
z(t)=y(t)-\sum_{i=1}^{k} p_{i}(t) y\left(r_{i}(t)\right) .
$$

Further, assuming that (1.7) and (1.6) hold, we define for $t \geq t_{1}$

$$
c(t)=\frac{(-1)^{n-1}}{(n-1) !} \int_{t}^{\infty}(s-t)^{n-1} u(s) H(y(h(s))) d s
$$

By assumptions (1.7) and (1.6), the above integral converges, thus $c(t)$ is a well defined real-valued function, and

$$
\lim _{t \rightarrow \infty} c(t)=0 .
$$

Note that the $n$th derivative of $c$ is $c^{(n)}(t)=-u(t) H(y(h(t)))$. For simplicity of notation, define

$$
w(t)=z(t)+c(t)-F(t),
$$

where $F^{(n)}(t)=f(t)$.

Lemma 3.5. Suppose that $p_{j}(t)$ satisfies the condition (1.15). Assume $r_{i}(t)=$ $t-\alpha$ where $i$ is as in (1.15) and $\alpha$ is a positive scalar. Let (1.2), (1.4)- (1.10) 
hold. Suppose that $y(t)$ is a non-oscillatory solutionof (1.1) with $z(t), c(t)$, and $w(t)$ as defined in (3.1), (3.7) and (3.9) respectively. If $y(t)>0$ in some interval $\left[t_{1} \infty\right)$ then either $\lim _{t \rightarrow \infty} w(t)=-\infty$ or $\lim _{t \rightarrow \infty} w(t)=\lambda$ (finite) and $\lim _{t \rightarrow \infty} w^{(j)}(t)=0, j=1,2, \ldots, n-1$ and $(-1)^{n+j} w^{(j)}(t)<0, j=1,2, \ldots, n-1$. If $y(t)<0$ in some interval $\left[t_{1} \infty\right)$ then either $\lim _{t \rightarrow \infty} w(t)=\infty$. or $\lim _{t \rightarrow \infty} w(t)=$ $\lambda$ (finite), and $\lim _{t \rightarrow \infty} w^{(j)}(t)=0, j=1,2, \ldots, n-1$ and $(-1)^{n+j} w^{(j)}(t)>0, j=$ $1,2, \ldots, n-1$.

Proof. Let $y(t)$ be an positive solution of (1.1) for $t \geq t_{1}>t_{0}$. Then for $t \geq t_{1}$, using (3.1), (3.7) and (3.9) in (1.1), we obtain

$$
w^{(n)}(t)=-v(t) G(y(g(t))) \leq 0 .
$$

Hence it follows from (3.10) that $w, w^{\prime}, \ldots, w^{(n-1)}$ are monotonic and of constant sign on some interval $\left[t_{1}, \infty\right)$. From (3.8) it follows, $c(t) \rightarrow 0$ as $t \rightarrow \infty$. Consequently,

$$
\lim _{t \rightarrow \infty} w(t)=\lim _{t \rightarrow \infty} z(t)=\lambda,
$$

where $\lambda$ is a finite real number or $\lambda=-\infty$ or $\lambda=+\infty$. The proof follows on similar lines as in [11][Lemma 2.4] with the following difference. The condition " $G$ is non decreasing "is used in [11] to prove

$$
\liminf G(y(g(t))) / t^{n^{*}}=0
$$

implies

$$
\left.\liminf _{t \rightarrow \infty} y(t)\right) / t^{n^{*}}=0 .
$$

In fact, if (3.12) holds then using (1.2) and (1.10), one may get lim inf $y(g(t)) / t^{n^{*}}=0$. Then from (1.9) and the fact that $\lim _{t \rightarrow \infty} g(t)=\infty$, it follows that (3.13) holds. Thus the decreasing condition on $G$ is relaxed. Since Lemma 2.5 which is applied to [11][Lemma 2.4] holds for both bounded and unbounded $v(t)$ but lemma 3.1, that would be applied to prove this lemma, does not hold for unbounded $y(t)$, therefore, $\lambda=0$ could not be achieved, and, that is why, the conclusion of this lemma is slightly different from that of [11].

Remark 3.6. The above Lemma holds if we assume (1.17) in stead of (1.15) subject to the condition that there exists $i \in\{1,2, \ldots, k\}$ such that $p_{i}(t) \geq 1$. Further, if $y$ is assumed to be a bounded solution in the above then the conclusion would follow, but the conditions (1.7) ad (1.10) are not required.

Remark 3.7. Lemma 3.5 generalizes and improves [11][Lemma 2.4] to higher order NDDE with several delay under derivative sign, and with, positive and negative, coefficients. Note that the condition that $G$ is non decreasing is assumed in [11][Lemma 2.4] unlike in lemma 3.5 of this article. 


\section{MAIN RESULTS-II}

In this section, we present the results to find sufficient conditions so that every non oscillatory solution of (1.1) tends to zero as $t \rightarrow \infty$.

Theorem 4.1. Let $n \geq 2$ be any positive integer. Suppose that, one of the conditions (1.12) or (1.13)hold. Assume for each $j, \liminf _{t \rightarrow \infty}\left|p_{j}(t)\right|>0$, in case (1.13) holds. If (1.2), (1.4), (1.6),(1.7), (1.9) (1.10)and (1.11) hold, then every non oscillatory solution of (1.1) tends to zero as $t \rightarrow \infty$.

Proof. Let $y(t)$ be a non-oscillatory solution of (1.1), which is eventually positive for $t \geq T_{1}$. With the assumptions (1.6) and (1.7) in hand, we set $z(t), c(t)$, and $w(t)$ as in (3.1), (3.7), and (3.9) respectively for $t \geq t_{0}>T_{1}$, to obtain (3.8) and (3.10), which implies $w(t), w^{\prime}(t), \ldots, w^{(n-1)}(t)$ are monotonic and of one sign for $t \geq t_{1} \geq t_{0}$. If possible, let $y(t)$ be unbounded. Then there exists a sequence $\left\{y\left(a_{k}\right)\right\}$ such that

$$
\lim _{k \rightarrow \infty} a_{k}=\infty, \lim _{k \rightarrow \infty} y\left(a_{k}\right)=\infty \text { and } y\left(a_{k}\right)=\max \left\{y(t): t_{1} \leq t \leq a_{k}\right\}
$$

From (1.4), we find a positive real $\gamma$ and a positive integer $n_{1}$ such that $F\left(a_{k}\right)<\gamma$ for $k>n_{1}$. By virtue of (3.8), for arbitrary positive $\epsilon$, it follows that $w\left(a_{k}\right) \geq$ $y\left(a_{k}\right)(1-p)-\epsilon-\gamma$ for the case when (1.12) holds or $w\left(a_{k}\right) \geq y\left(a_{k}\right)-\epsilon-\gamma$ for the case when (1.13)holds. In either case, we find $\lim _{k \rightarrow \infty} w\left(a_{k}\right)=\infty$, which, due to the monotonic nature of $w(t)$, implies $\lim _{t \rightarrow \infty} w(t)=\lambda=\infty$. This further implies $w(t)>0, w^{\prime}(t)>0$ for $t \geq t_{2} \geq t_{1}$. Since $w^{(n)}(t) \not \equiv 0$ and is negative, then because of Lemma 2.4, there exists a positive integer $m$ such that $n-m$ is odd and for $t \geq t_{3} \geq t_{2}$, we have $w^{(j)}(t)>0$ for $j=0,1, \ldots, m$ and $w^{(j)}(t) w^{(j+1)}(t)<0$ for $j=m, m+1, \ldots, n-2$. Clearly, $\lim _{t \rightarrow \infty} w^{(m)}(t)=l$ (finite) exists and $m \geq 1$. Integrating (3.10) $n-m$ times, we obtain

$$
w^{(m)}(t)=l+\frac{(-1)^{n-m-1}}{(n-m-1) !} \int_{t}^{\infty}(s-t)^{(n-m-1)} v(s) G(y(g(s))) d s .
$$

From which we get

$$
\int_{t}^{\infty}(s-t)^{(n-m-1)} v(s) G(y(g(s))) d s<\infty, \quad \text { for } t \geq t_{2},
$$

and then application of Lemma 2.4 yields

$$
\int_{t}^{\infty} s^{(n-m-1)} v(s) G(y(g(s))) d s<\infty .
$$


Because of (1.11), the inequality (4.3) implies $\liminf _{t \rightarrow \infty} \frac{y(t)}{t^{m-1}}=0$ and as $m \geq 1$, we find $B>0$, such that $w(t)>B t^{m-1}$ for $t \geq t_{3} \geq t_{2}$. Hence we obtain

$$
\liminf _{t \rightarrow \infty} \frac{y(t)}{w(t)}=0
$$

Setting,

$$
a_{j}(t)=p_{j}(t) \frac{w\left(r_{j}(t)\right)}{w(t)} \text { for } j=1,2, \ldots, k \text { and } t \geq t_{4}>t_{3},
$$

one finds $\lim _{t \rightarrow \infty} \frac{(F(t)-c(t))}{w(t)}=0$, by (1.5), (3.8) and $w(t)$ is increasing. Hence

$$
\begin{aligned}
& \lim _{t \rightarrow \infty}\left[\frac{y(t)}{w(t)}-\sum_{j=1}^{k} \frac{a_{j}(t) y\left(r_{j}(t)\right)}{w\left(r_{j}(t)\right)}\right]=\lim _{t \rightarrow \infty}\left[\frac{y(t)}{w(t)}-\sum_{j=1}^{k} \frac{a_{j}(t) y\left(r_{j}(t)\right)}{w\left(r_{j}(t)\right)}-\frac{(F(t)-c(t))}{w(t)}\right] \\
& =\lim _{t \rightarrow \infty}\left[\frac{y(t)-\sum_{j=1}^{k} p_{j}(t) y\left(r_{j}(t)\right)-(F(t)-c(t))}{w(t)}\right]=\lim _{t \rightarrow \infty}\left[\frac{w(t)}{w(t)}\right]=1
\end{aligned}
$$

Since $\frac{w\left(r_{j}(t)\right)}{w(t)}<1$ for each $j$, therefore, if $p_{j}(t)$ is defined as in (1.12) then $0 \leq$ $a_{j}(t)<p_{j}(t) \leq p_{j}<1$. On the other hand, if $p_{j}(t)$ is defined as in (1.13) then $0 \geq a_{j}(t) \geq p_{j}(t) \geq-b_{j}>-1$. As a result, it is evident that, for $j=1,2, \ldots, k$, if $p_{j}(t)$ satisfiy (1.12) or (1.13) then $a_{j}(t)$ also satisfiy the corresponding conditions of (1.12) or (1.13). If (1.12) holds then by using (4.4) and Lemma 3.1(a) we obtain

$$
\lim _{t \rightarrow \infty}\left[\frac{y(t)}{w(t)}-\sum_{j=1}^{k} \frac{a_{j}(t) y\left(r_{j}(t)\right)}{w\left(r_{j}(t)\right)}\right] \leq 0,
$$

which contradicts (4.5). However, if (1.13) holds, then by virtue of lemma 3.4, we obtain

$$
\lim _{t \rightarrow \infty}\left[\frac{y(t)}{w(t)}-\sum_{j=1}^{k} \frac{a_{j}(t) y\left(r_{j}(t)\right)}{w\left(r_{j}(t)\right)}\right]=0,
$$

a contradiction to (4.5). Hence $\{y(t)\}$ is bounded, this implies $w(t)$ is bounded. From (1.4), (1.5) and monotonic nature of $w(t)$, it follows that $\lim _{t \rightarrow \infty} w(t)=\lim _{t \rightarrow \infty} z(t)=$ $\lambda$ (finite). Next we prove that $\liminf _{t \rightarrow \infty} y(t)=0$. Integrating (3.10) $n$ times, we obtain

$$
w(t)=\lambda+\frac{(-1)^{n-1}}{(n-1) !} \int_{t}^{\infty}(s-t)^{(n-1)} v(s) G(y(g(s))) d s
$$

for $t \geq t_{1}$, where $t_{1}$ is some large positive integer. This implies

$$
\frac{1}{(n-1) !} \int_{t}^{\infty}(s-t)^{(n-1)} v(s) G(y(g(s))) d s<\infty, \quad t \geq t_{1} .
$$


The above inequality, together with Lemma 2.4 yields

$$
\int_{t}^{\infty} s^{n-1} v(s) G(y(g(s))) d s<\infty, \quad t \geq t_{1} .
$$

From the inequality (4.8), it follows due to (1.8) that $\liminf _{t \rightarrow \infty} G(y(g(t)))=0$. Since $\lim _{t \rightarrow \infty} g(t)=\infty$, it can be easily shown that $\liminf _{t \rightarrow \infty} G(y(t))=0$. Thus, $\liminf _{t \rightarrow \infty} y(t)=0$ due to (1.2), continuity of $G$ and boundedness of $y(t)$. Using Lemma 3.1, we conclude that $\lim _{t \rightarrow \infty} z(t)=0$ and $\lim _{t \rightarrow \infty} y(t)=0$. Thus, the conclusion of the theorem holds, for $y(t)>0$. If $y(t)$ is an eventually negative solution of (1.1), for large $t$, then setting $x(t)=-y(t)$, one has to obtain $x(t)$ as a positive solution of

$$
\left(x(t)-\sum_{j=1}^{k} p_{j}(t) x\left(r_{j}(t)\right)\right)+v(t) \tilde{G}(x(g(t)))-u(t) \tilde{H}(x(h(t)))=\tilde{f}(t)
$$

where

$$
\tilde{f}(t)=-f(t), \quad \tilde{G}(v)=-G(-v) \quad \tilde{H}(v)=-H(-v) .
$$

Further,

$$
\tilde{F}(t)=-F(t) \text { implies } \quad \tilde{F}^{n}(t)=\tilde{f}(t),
$$

and

$$
\text { (i) } x \tilde{G}(x)>0, x \tilde{H}(x)>0 \text { for } x \neq 0 \text {. (ii) } \tilde{H} \text { is bounded.(iii) } \tilde{G}(v) \geq \delta v, v>0
$$

hold. In view of the above facts, one may complete the proof by proceeding as in $y>0$ case.

Remark 4.2. Theorem 4.1 extends and generalizes [11][Theorem 3.6].

The following corollary follows from the theorem4.1.

Corollary 4.3. Every unbounded solution of (1.1) oscillates under the assumptions of Theorem 4.1.

Theorem 4.4. Let $n \geq 2$. Suppose that, $p_{j}(t)$ satisfies one of the conditions (1.12)-(1.15).Further suppose, that $x H(x)>0$. If (1.2), (1.4)-(1.6) and (1.8) hold, then every bounded solution of (1.1) which is non oscillatory tends to zero as $t \rightarrow \infty$.

Proof. The proof is similar to [3][Theorem 2.10]. 
Remark 4.5. The above theorem 4.4 holds when $G$ is linear, super linear, or sublinear and it improves, generalizes [11][theorem 3.5], which is concerned with NDDE(1.18). The improvement is due to the fact that the condition " $G$ is non decreasing" is relaxed in the theorem 4.4 .

Remark 4.6. The assumption (1.10) implies $G$ is linear or super linear. To deal with the unbounded solutions, we used (1.10) in Theorem 4.1, though we do not require this, or even (1.7) while dealing with bounded solutions in Theorem4.4.

Theorem 4.7. Suppose that $n \geq 2$, and that the condition (1.16) holds. Assume that $g\left(r_{j}(t)\right)=r_{j}(g(t))$, $\liminf _{t \rightarrow \infty}\left(r_{i}(t) / t\right)>0$ and $\liminf _{t \rightarrow \infty} r_{i}^{\prime}(t)>0$, for $j=1,2, \ldots, k$. Let (1.2), (1.4)-(1.7), (1.9) and (1.10) hold. Further assume the following conditions.

$$
\int_{t_{0}}^{\infty} t^{n-2} v^{*}(t) d t=\infty
$$

where $v^{*}(t)=\min \left\{v(t), v\left(r_{1}(t)\right), v\left(r_{2}(t)\right), \ldots, v\left(r_{k}(t)\right)\right\}$.

For $x_{i}>0, i=1,2, \ldots, k+1$ and $u>0$,

$G\left(\sum_{i=1}^{k+1} x_{i}\right) \leq \delta \sum_{i=1}^{k+1} G\left(x_{i}\right) \quad$ and $\quad G\left(u x_{i}\right) \leq G(u) G\left(x_{i}\right)$.

For $x_{i}<0, i=1,2, \ldots, k+1$, and $u>0$,

$G\left(\sum_{i=1}^{k+1} x_{i}\right) \leq \delta \sum_{i=1}^{k+1} G\left(x_{i}\right) \quad$ and $\quad G\left(u x_{i}\right) \leq G(u) G\left(x_{i}\right)$.

Then every eventually positive or eventually negative solution of (1.1) tends to zero as $t \rightarrow \infty$.

Proof. Let $y(t)$ be a solution of (1.1) for $t \geq t_{0} \geq T_{1}$, which is eventually positive - All we need to show is $\lim _{t \rightarrow \infty} y(t)=0$. Considering $z(t), c(t)$, and $w(t)$ as in (3.1), (3.7) and (3.9) respectively, we obtain (3.10) for $t>t_{1} \geq t_{0}$, which implies that $w(t), w^{\prime}(t), w^{(2)}(t), \ldots, w^{(n-1)}(t)$ are monotonic and of one sign for $t \geq t_{1}$. Clearly, (3.8) holds due to (1.6) and (1.7). Using (1.4), (1.5) and (3.8), we find that (3.11) holds i.e; $\lim _{t \rightarrow \infty} w(t)=\lim _{t \rightarrow \infty} z(t)=\lambda$, and $\lambda$ takes a value in the extended real number set. If $\lambda=0$, then $\lim _{t \rightarrow \infty} y(t)=0$ because $y(t) \leq z(t)$. If $\lambda<0$, then $z(t)<0$, for large $t$. However, $z(t) \geq 0$ by (1.16), a contradiction. If $\lambda>0$, then $w(t)>0$ for $t \geq t_{2}$. This, together with (3.10) and lemma 2.3, ensure that, there exists an integer $m$ such that $n-m$ is odd, $0 \leq m \leq n-1$ and $w^{(j)}(t)>0$ for $j=0,1, \ldots, m$ and $(-1)^{n+j-1} w^{(j)}(t)>0$ for $j=m+1, m+2, \ldots, n-1$. Then it follows that $\lim _{t \rightarrow \infty} w^{(m)}(t)=l$ exists and $\lim _{t \rightarrow \infty} w^{(i)}(t)=0$ for $i=m+1, m+2, \ldots, n-1$. If 
$0<\lambda<\infty$ then $m=0$, but $\lambda=\infty$ implies $m>0$ such that $n-m$ is odd. Integrating (3.10), $n-m$ times from $t$ to $\infty$ one gets (4.1). This implies (4.2).

Using Lemma 2.4, we obtain for $T_{2} \geq t_{3}$,

$$
\int_{T_{2}}^{\infty} s^{n-m-1} v(s) G(y(g(s))) d s<\infty .
$$

Note that,since each $r_{i}(t)$ is monotonic increasing,it's inverse function, $r_{i}^{-1}(t)$ exists for $i=1,2, \ldots, k$, such that $r_{i}^{-1}\left(r_{i}(t)\right)=t$. Since $v(t)>v^{*}\left(r_{i}^{-1}(t)\right)$,it follows that

$$
\int_{T_{2}}^{\infty} s^{n-m-1} v^{*}\left(r_{i}^{-1}(s)\right) G(y(g(s))) d s<\infty .
$$

As $G$ is continuous, and $0 \leq-p_{j}(t) \leq b_{j}, G\left(-p_{j}(t)\right)$ has an upperbound $\gamma>0$ Then substituting $s$ by $r_{i}(t)$ in the above in equation, and then multiplying by the scalar $\gamma$ we obtain

$$
\gamma \int_{T_{3}}^{\infty}\left(r_{i}(t)\right)^{n-m-1} v^{*}(t) G\left(y\left(g\left(r_{i}(t)\right)\right)\right) r_{i}^{\prime}(t) d t<\infty,
$$

where $T_{3} \geq r_{i}^{-1}\left(T_{2}\right)$. Since $\liminf _{t \rightarrow \infty} r_{i}^{\prime}(t)>0$ then,for $t \geq T_{3} \geq T_{2}, r_{i}^{\prime}(t)>\beta>0$. Further, $\liminf \inf _{t \rightarrow \infty}\left(r_{i}(t) / t\right)>0$ implies that we find $\alpha>0$ such that $r_{i}(t)>\alpha t$ for large $t$. Then using all this and the fact that $G\left(-p_{j}(g(t))\right) \leq \gamma$, we obtain

$$
\beta \alpha^{n-m-1} \int_{T_{3}}^{\infty} t^{n-m-1} v^{*}(t) G\left(-p_{j}(g(t))\right) G\left(y\left(g\left(r_{j}(t)\right)\right)\right) d t<\infty .
$$

This with the use of (4.14) yields

$$
\int_{T_{4}}^{\infty} t^{n-m-1} v^{*}(t) G\left(-p_{j}(g(t))\left(y\left(g\left(r_{j}(t)\right)\right)\right)\right) d t<\infty .
$$

Since $g\left(r_{j}(t)\right)=r_{j}(g(t))$, then the above inequality yields

$$
\int_{T_{4}}^{\infty} t^{n-m-1} v^{*}(t) G\left(-p_{j}(g(t))\left(y\left(r_{j}(g(t))\right)\right)\right) d t<\infty .
$$

The above inequation holds for $j=1,2, \ldots, k$. Hence addition of the above inequation for $j=1,2, \ldots, k$ yields

$$
\int_{T_{4}}^{\infty} t^{n-m-1} v^{*}(t) \sum_{j=1}^{k} G\left(-p_{j}(g(t))\left(y\left(r_{j}(g(t))\right)\right)\right) d t<\infty .
$$

From (4.16) and the fact that $v(t) \geq v^{*}(t)$, we obtain

$$
\int_{T_{4}}^{\infty} t^{n-m-1} v^{*}(t) G(y(g(t))) d t<\infty .
$$


Adding (4.18) and (4.19), and applying (4.14) we obtain

$$
\int_{T_{4}}^{\infty} t^{n-m-1} v^{*}(t) G(z(g(t))) d t<\infty .
$$

If $m=0$ then, from (4.13) and (4.20) it follows that $\liminf _{t \rightarrow \infty} t G(z(g(t)))=0$, which, with the application of (1.2), (1.10) and the assumption $\lim _{t \rightarrow \infty} g(t)=\infty$ yields : $\lim _{t \rightarrow \infty} z(t)=0$, a contradiction. If $m>0$ then there exists $M_{0}>0$ such that $w(t)>M_{0} t^{m-1}$ and by (1.5) and (3.8), we can find $0<M_{1}<M_{0}$ such that

$$
z(t)>M_{1} t^{m-1} \quad \text { for } t \geq T_{5} \geq T_{4} .
$$

Due to (1.9), we can find $b>0$ such that $g(t) / t>b>0$ for large $t$. Then using (4.21)and (1.10) one gets

$$
\begin{aligned}
\int_{T_{5}}^{\infty} t^{n-m-1} v^{*}(t) G(z(g(t))) d t & \geq \delta \int_{T_{5}}^{\infty} t^{n-m-1} v^{*}(t)(z(g(t))) d t \\
& \geq \delta M_{1} b^{m-1} \int_{T_{5}}^{\infty} v^{*}(t) t^{n-2} d t=\infty
\end{aligned}
$$

by (4.13), a contradiction due to (4.20). Hence the proof for the case $y(t)>0$ is complete. If $y(t)<0$, eventually for large $t$, then one may move with the substitution $x(t)=-y(t)$ as in the proof of the theorem 4.1 and note that, $x(t)$ is a positive solution of (1.1) with (4.10), (4.11) and (4.12). Then proceeding as above, in the proof for the case $y(t)>0$, and using (4.15) we prove that $\lim _{t \rightarrow \infty} x(t)=0$, which implies $\lim _{t \rightarrow \infty} y(t)=0$, and this, completes the proof of the theorem.

Remark 4.8. (i)The condition (1.16) is less restrictive than (1.14).

(ii) The above theorem 4.7 improves and generalizes [11][Theorem 3.7].

Remark 4.9. The function $G$ satisfying (1.2) (1.10), (4.14) and (4.15) could be $G(u)=$ $\left(\beta+|u|^{\mu}\right)|u|^{\lambda} \operatorname{sgn}(u)$, where $\lambda>0, \mu>0, \lambda+\mu \geq 1, \beta \geq 1$. To check the authenticity, one may use the inequality(see[2, p.292])

$$
u^{p}+v^{p} \geq \begin{cases}(u+v)^{p}, & 0 \leq p<1 \\ 2^{1-p}(u+v)^{p}, & p \geq 1\end{cases}
$$

Remark 4.10. For $n \geq 2$, the condition

$$
\int_{t_{1}}^{\infty} v^{*}(t)=\infty
$$

implies (1.3). Further the condition (4.22) implies (4.13) which further implies (1.11). 
Theorem 4.11. Suppose that $n \geq 2$, and that (1.16) holds. Assume that all assumptions in theorem 4.7, except (4.13), hold. Suppose that $v(t)$ is monotonic and (1.11) hold hold. Then every solution of (1.1) tends to zero as $t \rightarrow \infty$, if it does not oscillate.

Proof. All that, we need to show is (4.13) and (1.11) are equivalent, if $v(t)$ is monotonic. By remark 4.10, (4.13) implies (1.11). Therefore, it is enough to show that (1.11) implies (4.13), if $v(t)$ is monotonic. Clearly, if $v(t)$ is decreasing then $v^{*}(t)=v(t)$. Therefore, the equivalence of (1.11) and (4.13) is crystal clear. Next, if $v(t)$ is increasing then suppose that (1.11) holds for $t \geq t_{1}$, which implies $v^{*}(t)=v(r(t))$ where $r(t)=\min _{i}\left\{r_{i}(t)\right\}, t \geq t_{1}$, from which one easily gets, $r(t) \leq t$ and $r(t) \rightarrow \infty$ as $t \rightarrow \infty$. Note that, if $\liminf _{t \rightarrow \infty} r_{i}^{\prime}(t)>0$ for $i=1,2, \ldots, k$ then $\liminf _{t \rightarrow \infty} r^{\prime}(t)>0$. Let $\beta=\liminf _{t \rightarrow \infty} v(t)$. Hence for $T_{1} \geq t_{1}$, $\int_{T_{1}}^{\infty} t^{n-2} v^{*}(t) d t=\int_{T_{1}}^{\infty} t^{n-2} v(r(t)) d t \geq \beta \int_{T_{1}}^{\infty} t^{n-2} d t=\infty$ by (1.11). Thus, (4.13) holds and is equivalent to (1.11), if $v(t)$ is monotonic. The proof is complete.

Theorem 4.12. Suppose that $n$ is odd and $p_{j}(t)$ satisfies the condition (1.15). Let (1.2) and (1.6)-(1.10) hold. Consider the homogeneous NDDE

$$
\left(y(t)-\sum_{j=1}^{k} p_{j}(t) y\left(r_{j}(t)\right)\right)^{(n)}+v(t) G(y(g(t)))-u(t) H(y(h(t)))=0 .
$$

Then the following statements hold.

(i) If $y(t)$ is a solution of (4.23) and is bounded then it oscillates.

(ii)] If $y(t)$ is a non oscillatory solution of (4.23) and is unbounded then $\lim _{t \rightarrow \infty}$ $\left|\sum_{j=1}^{k} y\left(r_{j}(t)\right)\right|=\infty$ or $\liminf _{t \rightarrow \infty}|y(t)|=0$

Proof. Let us prove (A) and assume $y(t)$ to be a eventually positive and bounded solution of (4.23) in some interval $\left[t_{1} \infty\right)$. Then taking $c(t)$ as in (3.7), we obtain (3.8), due to (1.6). Then defining $z(t)$ as in (3.1) we obtain

$$
z^{(n)}(t)=-v(t) G(y(g(t))) \leq 0 .
$$

From this, it follows that $z(t), z^{\prime}(t), \ldots, z^{(m-1)}(t)$ are monotonic and of constant sign on some interval $\left[t_{1}, \infty\right)$. Then $\lim _{t \rightarrow \infty} z(t)=\lambda$ (finite) because $z$ is monotonic and bounded. Then integrating (4.24), $n$ times from $t$ to $\infty$, we obtain

$$
z(t)=\lambda+\frac{(-1)^{n-1}}{(n-1) !} \int_{t}^{\infty}(s-t)^{n-1} v(s) G(y(g(s))) d s,
$$

Consequently, (4.7) holds and then Lemma 2.4 yields (4.8). The inequality (4.8), due to $(1.8)$ yields 
$\liminf _{t \rightarrow \infty} G(y(g(t)))=0$. Since $\lim _{t \rightarrow \infty} g(t)=\infty$, it can be easily shown that $\liminf _{t \rightarrow \infty} G(y(t))=0$. This implies due to (1.2)and continuity of $G$ that $\liminf _{t \rightarrow \infty}$ $y(t)=0$. Since $n$ is odd then from lemma 3.5 and remark 3.6, it follows that $z^{\prime}(t)<0$ and $\lim _{t \rightarrow \infty} z(t)=\lambda$ (finite). Then by lemma 3.1 it follows that $\lambda=0$. Hence $z(t)>0$ in some interval $\left[t_{2} \infty\right)$. This implies $y(t)>\sum_{j=1}^{k} p_{j}(t) y\left(r_{j}(t)\right) \geq y\left(r_{i}(t)\right)$ as $p_{i}(t) \geq 1$ by (1.15). This yields $\lim \inf _{t \rightarrow \infty} y(t)>0$, a contradiction. Hence the bounded solution cannot be eventually positive. Following similar arguments, it can be shown that, a bounded solution cannot be eventually negative. Next let us proceed to prove (B) and consider $y(t)$ to be an eventually positive solution of (4.23) which is unbounded in some interval $\left(t_{1} \infty\right)$. Then using (1.6) and (1.7), $c(t)$ is set as in (3.7) and $z(t)$ as in (3.1), to get (4.24). Then by lemma 3.5 it follows either $\lim _{t \rightarrow \infty} z(t)=\lambda$ (finite) or $\lim _{t \rightarrow \infty} z(t)=-\infty$. If the latter holds then since $p_{j}(t)$ for $j=1,2, \ldots, k$ are bounded, there exists a positive scalar $b$ such that $0<p_{j}(t)<b$. From (3.1) it follows that

$$
\begin{aligned}
z(t) & =y(t)-\sum_{j+1}^{k} p_{j}(t) y\left(r_{j}(t)\right) \\
& \geq-b \sum_{j=1}^{k} y\left(r_{j}(t)\right) .
\end{aligned}
$$

This implies $\sum_{j=1}^{k} y\left(r_{j}(t)\right) \geq \frac{z(t)}{-b} \rightarrow+\infty$ as $t \rightarrow \infty$ So $\lim _{t \rightarrow \infty}\left|\sum_{j=1}^{k} y\left(r_{j}(t)\right)\right|=+\infty$. If the former holds then (4.25), (4.7) and (4.8) follows. Then using (1.2), and (1.8), one may obtain $\liminf _{t \rightarrow \infty} y(t)=0$. as in the proof of theorem 4.4. The proof for the case when $y(t)<0$ for $t \geq t_{0}$ is similar. Thus, the theorem is proved.

Remark 4.13. In the proof of part (i) in the above theorem we don't need $H$ is bounded.

Theorem 4.14. Suppose that $n$ is even and $p_{j}(t)$ satisfies the condition (1.15). Let (1.2),(1.4)— (1.10) hold. Then the following statements hold.

(i) If $y(t)$ is a non oscillatory solution of (4.23) and is bounded then it tends to zero as $t \rightarrow \infty$.

(ii) If $y(t)$ is a non oscillatory solution of (4.23) and is un bounded then $\lim _{t \rightarrow \infty}$ $\left|\sum_{j=1}^{k} y\left(r_{j}(t)\right)\right|=\infty$ or $\liminf _{t \rightarrow \infty}|y(t)|=0$

Proof. The proof is similar to the proof of the above theorem.

We proceed to prove a lemma which would be useful for the next theorem. 
Lemma 4.15. Let $n \geq 2$ be any positive integer. Assume that $p_{j}(t)$ satisfies the condition (1.17). Suppose that (1.2), (1.4)—(1.7), (1.9), (1.10)and (1.11) hold, Let $y(t)$ be an eventually positive solution of (1.1). Define $z(t), c(t), w(t)$ as in (3.1), (3.7) and (3.9) respectively. Then either $\lim _{t \rightarrow \infty} w(t)=-\infty$ or $\lim _{t \rightarrow \infty} w(t)=0$ and $(-1)^{(n+k)} w^{(k)}(t)<0$ for $k=0,1,2, \ldots, n-1$., for large $t$. If $y(t)$ be an eventually positive solution of (1.1) then either $\lim _{t \rightarrow \infty} w(t)=\infty$ or $\lim _{t \rightarrow \infty} w(t)=0$ and $(-1)^{(n+k)} w^{(k)}(t)>0$ for $k=0,1,2, \ldots, n-1 .$, for large $t$.

Proof. Since (1.11) implies (1.8), we proceed as in lemma 3.5 to obtain $-\infty \leq \lambda=$ $\lim _{t \rightarrow \infty} w(t) \leq \infty$. if $\lambda=\infty$, then proceed as in the proof of theorem 4.1 and set

$$
a_{j}(t)=p_{j}(t) \frac{w\left(r_{j}(t)\right)}{w(t)} \text { for } j=1,2, \ldots, k \text { and } t \geq t_{4} .
$$

to prove $\liminf _{t \rightarrow \infty} \frac{y(t)}{w(t)}=0$ as in (4.4). Since $p_{j}(t) \geq 0$, then $a_{j}(t) \geq 0$. As $y(t)>0$ and $w(t)>0$, therefore we have $\left[\frac{y(t)}{w(t)}-\sum_{j=1}^{k} \frac{a_{j}(t) y\left(r_{j}(t)\right)}{w\left(r_{j}(t)\right)}\right] \leq \frac{y(t)}{w(t)}$. Then

$$
\liminf _{t \rightarrow \infty}\left[\frac{y(t)}{w(t)}-\sum_{j=1}^{k} \frac{a_{j}(t) y\left(r_{j}(t)\right)}{w\left(r_{j}(t)\right)}\right] \leq \liminf _{t \rightarrow \infty} \frac{y(t)}{w(t)}=0 .
$$

Then continuing the proof as that of in theorem 4.1, we obtain

$$
\lim _{t \rightarrow \infty}\left[\frac{y(t)}{w(t)}-\sum_{j=1}^{k} \frac{a_{j}(t) y\left(r_{j}(t)\right)}{w\left(r_{j}(t)\right)}\right]=1
$$

as in (4.5), a contradiction due to (4.26). Hence, if $\lambda \neq-\infty$ then $y$ is bounded. Again proceeding as inthe proof of theorem 4.1, and applying lemma 3.1, we find $\lambda=0$. This implies $(-1)^{(n+k)} w^{(k)}(t)<0$ for $k=0,1,2, \ldots, n-1$, for large $t$. The proof for the case when $y(t)$ is eventually negative for lrge $t$ is similar.

Remark 4.16. The condition (1.17) is less restrictive than (1.15).

For our next result we need the following hypothesis.

For every sequence $\left\{\sigma_{i}\right\} \subset(0, \infty)$ such that $\lim _{i \rightarrow \infty} \sigma_{i}=\infty$ and for every $\gamma>0$ such that the intervvals $\left(\sigma_{i}-\gamma, \sigma_{i}+\gamma\right), i=1,2, \ldots$, are non overlapping, and

$$
\sum_{i=1}^{\infty} \int_{\sigma_{i}-\gamma}^{\sigma_{i}+\gamma} t^{n-2} v(t) d t=\infty
$$

or

$$
\sum_{i=1}^{\infty} \int_{\sigma_{i}-\gamma}^{\sigma_{i}+\gamma} t^{n-1} v(t) d t=\infty
$$


Remark 4.17. Note that (4.27) impliies (1.11) and (1.11) implies (1.8) for $n \geq 2$. Further, (4.27) implies (4.28) for $n \geq 2$.

Theorem 4.18. Suppose that $n \geq 2$ and $p_{j}(t)$ satisfies the condition (1.17). Let (1.2),(1.4)-(1.7),(1.10), (1.9),(1.11) and (4.28) hold. Then the following statements hold.

(i)If $y(t)$ is a non oscillatory solution of (1.1) and is bounded then it tends to zero as $t \rightarrow \infty$.

(ii) If $y(t)$ is a non oscillatory solution of (1.1) and is un bounded then $\lim _{t \rightarrow \infty}$ $\left|\sum_{j=1}^{k} y\left(r_{j}(t)\right)\right|=+\infty$.

Proof. Suppose $y(t)$ be a positive solution of (1.1). we set $z(t), c(t)$, and $w(t)$ as in (3.1), (3.7), and (3.9) respectively, to obtain (3.8) and (3.10). Hence $w(t), w^{\prime}(t), \ldots, w^{(n-1)}(t)$ are monotonic and of one sign for $t \geq t_{1} \geq t_{0}$. From lemma 4.15 it follows that, either $\lim _{t \rightarrow \infty} w(t)=-\infty$ or $\lim _{t \rightarrow \infty} w(t)=0$ and $(-1)^{(n+k)} w^{(k)}(t)<0$ for $k=0,1,2, \ldots, n-1$, for large $t$.

Let us prove (i) and assume $y(t)$ be an eventually positive solution of (1.1) which is bounded. Conseequently $w(t)$ is bounded and $\lim _{t \rightarrow \infty} w(t)=-\infty$ is ruled out. Then we have $\lim _{t \rightarrow \infty} w(t)=0$. Integrating (3.10) $n$ times, we obtain (4.6) and consequently, (4.7) and (4.8) holds for $t \geq t_{2} \geq t_{1}$. If $\limsup _{t \rightarrow \infty} y(t)=\alpha>0$ then there exists a sequence $\left\{t_{n}\right\}$ such that $y\left(t_{n}\right)>M>0$ for $n \geq N_{1}$. From the continuity of $y(t)$ it follows that there exists $\mu_{n}>0$ with $\liminf _{n \rightarrow \infty} \mu_{n}>0$ such that $y(t)>M$ for $t \in\left(t_{n}-\mu_{n}, t_{n}+\mu_{n}\right)$. Then choosing $n$ large enough such that $\mu_{n}>\mu>0$ for $n \geq N_{2}>N_{1}$, we obtain

$$
\begin{aligned}
\int_{t_{2}}^{\infty} s^{n-1} v(s) G(y(g(s))) d s & \geq \sum_{n=N_{2}}^{\infty} \int_{t_{n}-\mu_{n}+\sigma}^{t_{n}+\mu_{n}+\sigma} s^{n-1} v(s) G(y(g(s))) d s \\
& \geq \delta M \sum_{n=N_{2}}^{\infty} \int_{t_{n}-\mu+\sigma}^{t_{n}+\mu+\sigma} s^{n-1} v(s) d s
\end{aligned}
$$

Hence it follows from (4.28) that

$$
\int_{t_{2}}^{\infty} s^{n-1} v(s) G(y(g(s))) d s=\infty
$$

which contradicts (4.8). If $y(t)$ is eventually negative and bounded, the the proof is similar. Thus, (i) is proved. Next let us prove (ii) and assume $y(t)$ be an eventually positive solution of (1.1) which is unbounded.Then either $\lim _{t \rightarrow \infty} w(t)=-\infty$ or $\lim _{t \rightarrow \infty} w(t)=0$ and $(-1)^{(n+k)} w^{(k)}(t)<0$ for $k=0,1,2, \ldots, n-1$., for large $t$. 
If the latter holds then, we obtain (4.8) by integrating (3.10) $n$ times. Since $y(t)$ is unbounded, then there exists a sequence $\left\{t_{n}\right\}$ such that $y\left(t_{n}\right)>M>0$ for $n \geq N_{1}$. Proceeding as above, by using (4.27), we obtain (4.29) and this implies

$$
\int_{t_{2}}^{\infty} s^{n-1} v(s) G(y(g(s))) d s=\infty
$$

which contradicts (4.8). Hence the only possibility left is $\lim _{t \rightarrow \infty} w(t)=-\infty$. This implies $\lim _{t \rightarrow \infty} z(t)=-\infty$ by (3.8) and (1.5). From (3.1) and (1.17), it follows that

$$
\begin{aligned}
z(t) & =y(t)-\sum_{j+1}^{k} p_{j}(t) y\left(r_{j}(t)\right) \\
& \geq-b \sum_{j=1}^{k} y\left(r_{j}(t)\right) .
\end{aligned}
$$

This implies $\sum_{j=1}^{k} y\left(r_{j}(t)\right) \geq \frac{z(t)}{-b} \rightarrow+\infty$ as $t \rightarrow \infty$

The proof for the case, $y(t)$ is eventually negative and unbounded for large $t$ is similar. Thus, $\lim _{t \rightarrow \infty}\left|\sum_{j=1}^{k} y\left(r_{j}(t)\right)\right|=+\infty$.

Remark 4.19. As (4.27) impliies the conditions (1.11) and (4.28), hence the above theorem holds if (4.27) is assumed in place of (1.11) and (4.28).

Remark 4.20. The above theorem 4.18 improves [12][Theorem 2.9] and generalize [10][Theorem 2.2].

The following examples enhance the significance of our results.

Example 4.21. The neutral differential equation

$$
(y(t)-4 y(t-\pi)-2 y(t-2 \pi))^{(3)}+2 y(t-3 \pi / 2)-y(t-\pi / 2)=0,
$$

satisfies all the conditions of Theorem4.12 and 4.18, with $p_{j}(t)$ satisfying the condition (1.15) and (1.17). As such, this neutral equation has a bounded oscillatory solution $y=\sin (t)$.

Example 4.22. The neutral differential equation

$$
\left(y(t)-\frac{1}{2 e} y(t-1)-\frac{1}{2 e^{2}} y(t-2)\right)^{(n)}+y^{3}(t-3)-e^{-2 t+6} y(t-3)=0,
$$

satisfies all the conditions of Theorem4.1, 4.4 and 4.18with $p_{j}(t)$ satisfying the condition (1.12) and (1.17). As such, this neutral equation has an bounded solution $e^{-t}$, which $\rightarrow 0$ as $t \rightarrow \infty$. 
Example 4.23. The neutral differential equation $\left(y(t)-\frac{1}{2} e^{-1} y(t-1)-\frac{1}{2} e^{-4} y(t-4)\right)^{(n)}+\frac{1}{t^{n-1}} y(t-2)-e^{-t} H(y(t-2))=\frac{e^{-t+2}}{t^{n-1}}-\frac{e^{2}}{\left(e^{2 t}+e^{4}\right)}$, $n \geq 2$ and $H(u)=u /\left(u^{2}+1\right)$, satisfies all the conditions of Theorems 4.1, 4.4 and 4.18 for $p_{j}(t)$ satidfying (1.12) and (1.17). Hence by the theorem, all bounded solutions oscillate or tend to zero as $t \rightarrow \infty$, as such $y(t)=e^{-t}$ is a solution of the above equation, which tends to zero as $t \rightarrow \infty$. Comparing the above equation to (1.19), one finds $\sigma=\alpha=2$, and so, neither ' $\sigma>\alpha$ nor $\alpha>\sigma$ 'holds. As such, the results of the papers $[6,7,8,13]$ fail to be applied to this equation.

\section{CONCLUSION}

It is to be marked that none of the results available so far in literature could be applied to the neutral equations given in the examples above. The theorems 4.7 and 4.18 of this article, for the particular case $k=1$ answer the open problem [1][problem 10.10.2, page 287] which says "extend the results of section 10.4 to equations where $p(t)$ does not lie in any of the following ranges."

$$
\begin{aligned}
& \text { (B1) } 1 \leq p(t) \leq p_{1}, \quad\left(\text { B2) } 0 \leq p(t) \leq p_{2}<1, \quad(\text { B3 })-1<-p_{3} \leq p(t) \leq 0,\right. \\
& \text { (B4) } p(t) \equiv-1, \quad(\text { B5 })<p(t) \leq 1 .
\end{aligned}
$$

This is because the range of $p(t)$ in the particular case of $k=1$ for Theorem 4.7 and that of Theorem 4.18 are found to be $-b \leq p(t) \leq 0$ and $0 \leq p(t) \leq b$ by (1.16) and (1.17) respectively.

\section{REFERENCES}

[1] I. Gyori and G. Ladas; Oscillation Theory of Delay-Differential Equations with Applications, Clarendon Press, Oxford, 1991.

[2] T. H. Hilderbrandt,Introduction to the Theory of Integration, Academic Press, New-York, 1963.

[3] B. Karpuz, R. N. Rath and L. N. Padhy, On Oscillation and asymptotic behaviour of a higher order neutral differential equation with positive and negative coefficients, Electro. J. Diff. Eqs., 2008 (2008), No. 113, pp. 1-15. MR2430910

[4] G. S. Ladde, V. Lakshmikantham and B. G. Zhang: Oscillation Theory of Differential Equations with Deviating Arguments, Marcel Dekker Inc.,New York, 1987. 
[5] S.C. Malik and S. Arora, "Mathematical Analysis", New Age International (p) Ltd. Publishers New Delhi, 2001.

[6] J. Manojlović, Y. Shoukaku, T. Tanigawa, N. Yoshida: Oscillation criteria for second order differential equations with positive and negative coefficients. Appl. Math. Comput., 181 (2006), No. 2, 853-863. Zbl 1110.34046

[7] Ozkan Ocalan,Oscillation of neutral differential equation with positive and negative coefficients,J. Math. Anal. Appl.,331 (2007),644-654.

[8] N. Parhi and S. Chand, Oscillation of second order neutral delay differential equations with positive and negative coefficients, J.Ind.Math.Soc. 66(1999),227-235.

[9] N. Parhi and R. N. Rath ; Oscillation criteria for forced first order neutral differential equations with variable coefficients, J. Math. Anal. Appl. 256 (2001),525-541.

[10] N. Parhi and R. N. Rath : On oscillation and asymptotic behaviour of solutions of forced first order neutral differential equations, Proceedings of Indian Acad. Sci. Math. Sci. 111 (2001), 337-350.

[11] N. Parhi and R. N. Rath : On oscillation of solutions of forced non linear neutral differential equations of higher order, Czech. Math. Journal, 53 (2003), 805-825 . MR2018832(2005g:34163)

[12] N. Parhi and R. N. Rath, On Oscillation of solutions of forced nonlinear neutral differential equations of higher order II, Annales Polonici Math. 81(2003), 101-110.

[13] R. N. Rath and N. Misra, Necessary and sufficient conditions for oscillatory behavior of solutions of a forced non linear neutral equation of first order with positive and negative coefficients, Math. Slovaca, 54 (2004), 255-266.

[14] Radhanath Rath, Chittaranjan Behera; Oscillatory and Asymptotic Behavior of a First order Neutral Equation of Discrete type with Variable Several Delay under $\Delta$ sign, International journal of mathematics and mathematical sciences, Hindawi publishing house, vol. 2018, article ID 4586176, https//doi.org $10.1155 / 20184586176$.

[15] R. N. Rath, K.C.Panda, S.K.Rath; Oscillatory behaviour of a first order neutral differential equation in relation to an old open problem To appear in IJMMS, Hindawi publishing House. 
[16] H. L. Royden, “ Real Analysis“, MacMilan Publ. Co. (3rd edition) New York, 1989.

[17] Y.Sahiner and A.Zafer,Bounded oscillation of non-linear neutral differential equations of arbitrary order,Czech.Math.J., 51 (126)(2001),185-195. 